\title{
Patterns of maternal feeding and child eating associated with eating disorders in the Norwegian Mother and Child Cohort Study (MoBa)
}

\author{
Lauren Reba-Harreleson, M.A. ${ }^{a}, b$, Ann Von Holle, M.S. ${ }^{a}$, Robert M. Hamer, Ph.D. ${ }^{a}, \mathrm{e}$, Leila \\ Torgersen, Ph.D.C, Ted Reichborn-Kjennerud, M.D.C,f, and Cynthia M. Bulik, Ph.D. a,b,d \\ aDepartment of Psychiatry, University of North Carolina at Chapel Hill First Floor, Neurosciences \\ Hospital, Campus Box \#7160, Chapel Hill, NC 27599-7160
}

bDepartment of Psychology, University of North Carolina at Chapel Hill CB\#3270 Davie Hall, Chapel Hill, NC 27599-3270

'Division of Mental Health, Norwegian Institute of Public Health, P.O.Box 4404 Nydalen, N-0403, Oslo, Norway.

dDepartment of Nutrition, University of North Carolina at Chapel Hill CB \#7461, Chapel Hill, NC 27599-7461

eDepartment of Biostatistics, University of North Carolina at Chapel Hill CB \#7420, Chapel Hill, NC 27599-7420

'Department of Epidemiology, Columbia University, New York, NY, USA

\section{Abstract}

The impact of eating disorders on maternal feeding practices and children's eating behaviors is not well understood. In the prospective Norwegian Mother and Child Cohort Study (MoBa),we compared self-reported feeding behavior in mothers with anorexia nervosa (AN), bulimia nervosa (BN), binge eating disorder (BED), and no eating disorders (No ED) as well as child eating behaviors and psychological symptoms. The sample was comprised of 13006 women and their children from a prospective population-based study of 100,000 births throughout Norway. Eating disorder status was measured 6 months prior to pregnancy and during pregnancy. Maternal feeding, child eating, and psychological variables were reported by mothers when their child was 36 months old. Mothers with $\mathrm{BN}$ and BED were more likely to report restrictive feeding styles and infant eating problems than mothers without eating disorders. Regarding pressured feeding style, no significant differences emerged across groups. Differences in self-reported feeding styles and children's eating behavior exist between mothers with and without eating disorders. Longitudinal follow-up will assist with determining the implications of feeding style on later growth trajectories and development.

(C) 2009 Elsevier Ltd. All rights reserved.

Address reprint requests and correspondence: Dr. Cynthia Bulik, Department of Psychiatry, University of North Carolina at Chapel Hill, CB \#7160, 101 Manning Drive, Chapel Hill, NC 27599-7160, USA. Phone: (919) 843 1689; Fax: (919) 966-5628, cbulik@med.unc.edu.

Publisher's Disclaimer: This is a PDF file of an unedited manuscript that has been accepted for publication. As a service to our customers we are providing this early version of the manuscript. The manuscript will undergo copyediting, typesetting, and review of the resulting proof before it is published in its final citable form. Please note that during the production process errors may be discovered which could affect the content, and all legal disclaimers that apply to the journal pertain. 


\section{Keywords}

Eating disorders; anorexia nervosa; bulimia; binge eating; mothers; child psychology

\section{Introduction}

Children of mothers with eating disorders may comprise an at-risk group for the potential development of eating disorder symptomatology (Patel, Wheatcroft, Park, \& Stein, 2002; Stein, et al., 2006). Although we know that eating disorders run in families, and that genetics play a role in this familial aggregation (Bulik, Hebebrand, et al., 2007; Bulik, Sullivan, \& Kendler, 2003; Klump, Burt, McGue, \& Iacono, 2007), we also know that environmental factors exert an influence on the development of eating disorders (Barr, et al., 2004; Johnson, Cohen, Kasen, \& Brook, 2002; Klump, Miller, Keel, McGue, \& Iacono, 2001; Reichborn-Kjennerud, Bulik, Tambs, \& Harris, 2004). Mothers with histories of eating disorders express concern over their ability to feed and parent their children appropriately (Franzen \& Gerlinghoff, 1997; Russell, Treasure, \& Eisler, 1998) and express considerable concern about whether their children are at risk for developing eating disorders themselves (Russell, et al., 1998). As an initial step in addressing their concerns, we sought to understand differences in maternal feeding styles and child eating behavior in women with eating disorders versus those with No ED.

The scant literature examining the impact of maternal eating disorder status on feeding styles and eating behavior in early childhood, suggests that, compared with healthy mothers, mothers with eating disorders tend to display more unregulated feeding and concern about their daughters' weight (from age 2 and up) (Agras, Hammer, \& McNicholas, 1999), regulate their children's eating behavior for fear of overeating (Russell, et al., 1998), show greater rigidity at mealtimes (Evans \& le Grange, 1995), and make attempts to help their young children lose weight (Agras, et al., 1999; Lacey \& Smith, 1987). One of the few prospective studies to date revealed that 10 year olds of mothers with eating disorders exhibit more dietary restraint and place greater value on weight/shape in their self-evaluation than controls (Stein, et al., 2006). Although the aforementioned studies are important in providing a preliminary understanding of maternal feeding and child eating behaviors in women with eating disorders and their offspring, they have been limited by small sample sizes, inability to compare across eating disorder subtypes, and they remain without replication. There have been no prior large-scale, prospective, population-based studies exploring the impact of eating disorder subtype on maternal feeding habits and child eating behaviors and psychological symptoms in young children.

The current study explored the impact of broadly defined eating disorders on maternal feeding practices and children's eating behaviors and psychological symptoms in a sample of 13,006 births in Norway. The present study's aims were threefold: (1) to compare maternally-reported feeding attitudes and practices in mothers with broadly defined eating disorders across diagnostic subtypes [anorexia nervosa (AN), bulimia nervosa (BN) and binge eating disorder (BED)] with mothers without eating disorders (No ED); (2) to compare maternally-reported eating behaviors and psychological symptoms (i.e., depression, anxiety, and obsessivecompulsive symptoms) in 36 month old children of mothers with eating disorders across diagnostic subtypes with children of mothers with No ED; (3) to examine the relation between maternally reported feeding behaviors and infant eating behavior in 36 month old children. 


\section{Material and Methods}

\subsection{The Study Sample}

The Norwegian Mother and Child Cohort Study ("Den norske mor og barn undersøkelsen" $\mathrm{MoBa}$ ) is a prospective population-based pregnancy cohort of 100000 births throughout Norway sponsored by the the Norwegian Institute of Public Health. Recruitment began in 1999 in all parts of Norway, and as of April, 2008, the total number of pregnancies enrolled was 100 349 (or 86007 mothers). Using lists of scheduled appointments at ultrasound labs, MoBa recruits women around two weeks before the routinely performed ultrasound examination (c. 17 th week) in pregnancy. Interested women receive an information folder, complete informed consent, and then receive two questionnaires on diet and health history. The participants also complete a questionnaire at 30 weeks gestation and with regular intervals after birth (see below). The MoBa cohort is linked to the Medical Birth Registry of Norway (MBRN), including data on all births after 16 weeks of pregnancy. This resource allows comparisons between participants and non-participants and suggests that MoBa participants tend to be somewhat older (mean 29.2 years versus 28.4 ) and better educated (56\% completed more than 13 years of school versus $33 \%$ ) than the general population. Rigorous analyses were conducted to identify participation bias in the MoBa (Magnus, et al., 2006). At the time of the current study, $42 \%$ of invited mothers participated. Although the response rate is somewhat low, it is not unusual for large studies and is not necessarily associated with sample bias (Hartge, 2006).

Initially, the analysis population for the current study included all pregnancies with clean data from MoBa Questionnaire 6 (36 months after birth, $\mathrm{N}=15$ 527), Questionnaire 1 (17 weeks, $\mathrm{N}=51$ 048), and the MBRN ( $\mathrm{N}=63$ 182). After merging these datasets, participants without valid values for demographic and response variables of interest were excluded, leaving 14198 participants. Finally, after accounting for missing data in Questionnaire 1, relevant to determining eating disorder diagnosis, 13006 mothers remained. If a woman had more than one pregnancy during the study period, only the first pregnancy in the study period is included in this analysis. The drop off between completed Questionnaire 1 and completed Questionnaire 6 above is not only due to attrition, but due primarily to the fact that many of the children on whom Questionnaire 1 was available had not yet reached the age at which Questionnaire 6 is administered. The first two questionnaires were completed by $94 \%$ of women who consented to participate. For the 30 weeks' gestation questionnaire, $92 \%$ were completed and returned. Finally, $88 \%$ of mothers completed and returned the 6 month questionnaire, $77 \%$ percent completed the 18 month questionnaire, and $61.4 \%$ completed the 36 month questionnaire.

\subsection{Assessments}

The assessments from the MoBa occurred in five waves: at 17 weeks prenatal, 30 weeks prenatal, 6 months, 18 months, and 36 months after child birth. Data were also available from the MBRN. The current study used assessment data relating to maternal feeding styles, child eating behaviors, psychological symptoms, and food allergies from the questionnaire sent at 36 months. We also used demographic data from the MBRN. Queried for the periods of 6 months prior to pregnancy or during pregnancy, eating disorder status was taken from diagnostic questions asked in the 17 weeks prenatal assessment.

2.2.1 Eating disorders and related behaviors-In addition to height and weight, all women are asked diagnostic questions relevant to eating disorders that were taken from the Norwegian Twin Panel assessment of eating disorders. These questions have been used widely in twin studies (Reichborn-Kjennerud, et al., 2003; Reichborn-Kjennerud, et al., 2004) and yield replicated prevalence estimates. Diagnostic algorithms were constructed from the 17 week prenatal questionnaire items to define the presence of broadly defined AN (amenorrhea 
not required), broadly defined $\mathrm{BN}$ at least weekly frequency of binge eating and purging), and broadly defined BED (at least weekly frequency of binge eating). Nine individuals with eating disorders not otherwise specified were excluded from the analyses due to lack of sufficient power to detect significant differences (Bulik, Von Holle, et al., 2007). AN was assessed before pregnancy only due to practical difficulties in determining low weight in the presence of pregnancy-related weight gain. BN and BED were assessed for both 6 months prior to pregnancy (retrospective assessment) and at the time of survey completion. Self reported weight and height were used to calculate pre-pregnancy body mass index (BMI, $\mathrm{kg} / \mathrm{m}^{2}$ ) and $\mathrm{BMI}$ at the time of assessment. Respondents were specifically asked to distinguish between pregnancy-related vomiting and self-induced vomiting as a purging method. Respondents completed questionnaire 1 at a median of 18.1 weeks gestation with inter-quartile range of 16.7-20.1 weeks, and range 5.3-41.9 weeks. Before or during pregnancy, BED and BN are mutually exclusive diagnoses as determined by the classification algorithm. The category BN included individuals who responded to questions that defined non-purging BN (i.e. fasting and exercise), purging BN (i.e. laxatives and vomiting), and individuals who could not reliably be categorized as purging or non-purging due to missing data.

To establish these diagnostic categories, a hierarchical algorithm was created to avoid overlap if individuals endorsed multiple disorders at different time points (e.g., AN before pregnancy and BN during pregnancy). The hierarchy's order of group assignment is as follows: AN, BN, BED, and No ED. Greater detail about the diagnostic hierarchy can be found elsewhere (Bulik, Von Holle, et al., 2007).

The MBRN form includes a variety of items that assess pregnancy outcome. The current study used maternal age, marital status, total number of previous live births, maternal education, child sex, birth weight, Apgar scores, gestational length, and gestational age.

2.2.2 Maternal feeding-From the 36 month questionnaire, the study used measurement of maternal feeding practices derived from the Child Feeding Questionnaire (Birch, et al., 2001), an instrument for assessing parental control in child feeding. Specifically, Restriction and Pressure to Eat subscales were used for the analyses, and were the only subscales available in the MoBa questionnaires. Validated by Birch et al. (2001), Pressure to Eat is a 4-item scale that measures the degree to which the mother encourages the child to eat by behaviors such as insisting that the child eat everything on his or her plate. The questions are as follows: (1) My child should always eat all the food on her plate, (2) I have to be especially careful to make sure my child eats enough, (3) If my child says, "I'm not hungry", I try to get him/her to eat anyway, (4) If I did not regulate my child's eating, she would eat much less than she should. The Restriction subscale has been validated using an 8-item scale (2001), for which the 36 month questionnaire offers all 8 items. The Restriction subscale statements are as follows: (1) I have to be sure that my child does not eat too many sweets (candy, ice cream, cake or pastries), (2) I have to be sure that my child does not eat too many high fat foods, (3) I have to be sure that my child does not eat too much of his/her favorite foods, (4) I intentionally keep some foods out of my child's reach, (5) I offer sweets (candy, ice cream, cake, pastries) to my child as a reward for good behavior, (6) I offer my child his/her favorite foods in exchange for good behavior, (7) If I did not guide or regulate my child's eating he/she would eat too many junk foods, and (8) If I did not guide or regulate my child's eating he/she would eat too much of his/her favorite foods. All items are scored from 1 (low restriction/pressure to eat) to 5 (high restriction/pressure to eat).

2.2.3 Child eating problems-Using the 36 month questionnaire, the study measured child eating habits, including items relating to: (1) not eating well, (2) having stomach aches or cramps, (3) vomiting without medical cause, and (4) not enjoying eating. Measurement of eating problems was taken from the CBCL - Child Behavior Checklist (Achenbach, 1991), 
internationally the most widely used instrument to assess early behavioral problems in children (and toddlers). Each item is scored from 1 (Not True) to 5 (Very True). Food allergies (present or absent) were obtained from maternal report in the 36 months questionnaire.

2.2.4 Child growth, development, and psychological symptoms-From the 36 month questionnaire, the current study used the child age, weight, and length measurements to compare to population norms of weight-for-age at 36 months using an algorithm provided by the Centers for Disease Control (2005). From the 36 month assessment, child anxiety, depressive, and obsessive-compulsive symptoms were examined. Questions regarding anxiety were from the CBCL (Achenbach, 1991). The anxiety subscale includes items relating to: (1) the child being too dependent, (2) getting too upset when separated from parents, and (3) being too fearful or anxious. Measurement of depressive and obsessive compulsive-type traits were taken from the ITSEA - Infant Toddler Social Emotional Assessment (Carter, Little, BriggsGowan, \& Kogan, 1999). The depressive subscale includes items regarding: (1) lack of energy, (2) having "less fun than other children," and (3) seeming "very unhappy, sad or depressed." The OCD-type behaviors are part of the atypical index and general anxiety subscale of the ITSEA (Carter, Briggs-Gowan, Jones, \& Little, 2003), and included: (1) worry about getting dirty, (2) needing things to be clean or neat, and (3) displaying repetitive ordering behavior. All items are scored from 1 (Not True) to 5 (Very True). This widely used measure covers phenomena of importance for early developmental problems and is not overlapping with questions found on the CBCL.

\subsection{Ethics}

The current study is a subproject of the MoBa (15), and has been approved by the appropriate regional committees for ethics in medical research and the Norwegian National Data Inspectorate. Approval was also obtained from the UNC Biomedical Institutional Review Board. Data transfer occurred via encrypted files over the internet and via physical transfer of storage media. No identifying information was handled in the current study. The team of UNC researchers was responsible for transporting data from Norway to the US electronically; the team in Norway was responsible for the statistical programming tasks necessary to prepare the data for transport.

\subsection{Statistics}

Data analysis was performed using the software packages, SAS/STAT ${ }^{\circledR}$ software, version 9.1 (SAS Institute Inc., 2000-2004) and JMP (SAS Institute Inc., 1994). The proportion of missing data across assessments ranges from $<1-2 \%$. Nonetheless, some missing data existed. Using JMP (SAS Institute Inc., 1994), missing data or impossible values were examined by performing range and value checking for all key variables. Range checks were conducted for all variables.

SAS/STAT ${ }^{\circledR}$ software, version 9.1 (SAS Institute Inc., 2000-2004) was used to perform statistical analyses for each aim. The response variables for aim one included maternal feeding practices (restrictive feeding and pressure to eat). For aim two, response variables included child feeding habits, child anxiety, depressive, and obsessive-compulsive symptoms. Child weight-for-age status at 36 months and child food allergies (present or absent) were added to the model as covariates to prevent confounding for reported child eating behavior related to child overweight or food allergies.

First, comparisons were made across the eating disorder groups on a small number of sociodemographic characteristics to ensure that the groups did not differ appreciably. Next, for each aim, all possible pair-wise comparisons were conducted between the four groups using Tukey's honest significant difference (HSD) post hoc test to explore group differences at the 
significance level of 0.05 . For the continuous response variables, ANOVA was used to describe the relation between eating disorder status and each predictor variable. For aim three, the examination of the relation between maternal reported feeding behaviors and child eating behavior, models were created corresponding to restrictive feeding and pressure to eat. ANOVAs were used to describe the relation between feeding behaviors and the predictor variable, child eating problems. Child weight-for-age and child food allergy status were added to each model as covariates to prevent confounding for feeding behavior related to child overweight or food allergy.

G*Power3 (Faul, Erdfelder, Lang, \& Buchner, in press) was used for power and sample size calculations. We computed power and sample size for a "small" effect size, defined as $\mathrm{d}=0.20$, in the context of a one-way, four-group ANOVA (Cohen, 1988). Assuming alpha $=0.05$, and a total sample size of 13006 , with available sample sizes of those with AN, BN, BED, and No $\mathrm{ED}$, we found $>90 \%$ statistical power would be achieved for $\mathrm{d}=0.20$. Thus, for these analyses, the sample was sufficiently powered to reject the null hypothesis for small effect sizes.

\section{Results}

Characteristics of all participating mothers are found in Table 1. Consistent with initial reports on the MoBa sample (Bulik, Von Holle, et al., 2007;Magnus, et al., 2006), the majority of the women were between the ages of 25-34, approximately $95 \%$ were married or cohabitating, and individuals were relatively well educated with approximately $77 \%$ attending junior college or higher.

\subsection{Prevalence of Eating Disorders and Associated Characteristics}

Seventeen women (0.13\%) met criteria for AN, $98(0.75 \%)$ for BN, $634(5 \%)$ for BED, and 12257 (94\%) for No ED. Significant differences emerged for maternal age at childbirth, parity, maternal pre-pregnancy BMI, and educational status across groups. Mothers with AN had a lower age at childbirth than all other groups. Women with BED had significantly more children than women without eating disorders. Mothers with AN had lower mean BMIs than all other groups; those with BED had significantly higher mean BMIs than those with AN and No ED. Mothers with BED were more likely to be less educated than those in the No ED group.

Characteristics of the offspring are found in Table 2. Offspring did not differ appreciably on demographic characteristics. The mean gestational age fell in the category of normal gestation (average gestational age approximately 280 days) (O'Reilly, 2007). Mean Apgar scores met healthy criteria (>7) for the 1 minute Apgar test (Finster \& Wood, 2005).

\subsection{Maternal Report of Restrictive Feeding and Pressure to Eat}

Total sample size, adjusted means, F, and $\mathrm{R}^{2}$ values for each analysis are reported in Table 3. From this point on, all p-values reported in the text for comparisons across eating disorder groups are Tukey-Kramer adjusted values. Adjusting for food allergy and child weight status, measures of restrictive feeding differed significantly across groups, whereas pressured feeding did not significantly differ across groups. In addition to the significant differences, we also present the magnitude of mean differences across groups given the highly unbalanced group sizes. Although statistical power was lower for the small AN group, a full appreciation of the results requires a discussion of mean differences as well as significance tests. Further, because certain means values change after adjusting for child weight status and food allergies, we report unadjusted means and the sample size for each analysis in Table 4.

Mothers with BN and BED both reported significantly higher levels of restrictive feeding than mothers in the No ED group, $p<.0354$ and $p<0.001$, respectively. Food allergy, $\mathrm{F}(1,9808)$ 
$=8.13, p<.005$, and weight-for-age at 36 months, $\mathrm{F}(1,9808)=72.96, p<.0001$, also significantly differed across eating disorders groups. Compared to No ED mothers $(\mathrm{M}=2.40$, $\mathrm{SE}=0.02$ ), adjusted mean scores of mothers with $\mathrm{BN}$ and BED were 0.24 and 0.18 higher, respectively. Although not significant, the adjusted mean score of mothers with AN was 0.30 lower than the No ED group, suggesting that they were less likely to engage in restrictive feeding than non-eating disordered mothers.

Regarding pressured feeding, there were no significant differences between mothers with eating disorders and those without, $\mathrm{F}(5,9793)=1.04, p=\mathrm{ns}$. Again, exploring means for the AN group, although not significant, their least square mean score was 0.22 and 0.20 lower than women with BN and BED respectively, and 0.14 lower than the No ED group (M = 2.47, SD $=0.89$ ), indicating that they reported less pressured feeding behavior. Overall, the mean differences actually suggest different feeding styles in women with AN than the referent, often in the opposite direction of women with BN and BED; however, the unbalanced sample sizes present in this sample decrease power to detect significant differences. Again, weight-for-age at 36 months, $\mathrm{F}(1,9793)=343.46, p<.0001$, was significantly higher across eating disorders groups.

To assess the impact of maternal weight status on reported restrictive feeding and pressure to eat, maternal BMI at 36 months was added as covariate to each model. Whereas maternal BMI had a significant effect as an independent predictor of the response $(p<0.001)$, it did not alter the significance or magnitude of the relation between eating disorder status and feeding style in any of the analyses.

\subsection{Maternal Report of Child Eating Behavior and Psychiatric Symptomatology}

Adjusted means for each analysis are reported in Table 3 and unadjusted means are reported in Table 4.

3.3.1 Child eating problems-Adjusting for food allergy and child weight status, reported child eating problems significantly differed across eating disorders group. Mothers with BN and BED reported higher levels of disordered eating behaviors in their children than mothers in the No ED group, $p<.03$ and $p<.003$, respectively. Food allergy, $\mathrm{F}(1,9817)=5.53, p<$. 02 and weight-for-age at 36 months, $\mathrm{F}(1,9817)=344.06, p<.0001$, also significantly differed across eating disorders groups. Again, exploring direction of effects and adjusted means, whereas both BN and BED mothers reported elevated scores .09 and .05 higher than the No ED group respectively, mean scores in mothers with AN were 0.09 lower than the referent (M $=1.29, \mathrm{SE}=0.007$ ), although nonsignificant.

3.3.2 Psychiatric symptoms-Reported infant anxiety and OCD significantly differed across eating disorder status. Mothers with BN and BED reported higher levels of anxiety symptoms in their children than mothers with No ED, $p<.03$ and $p<.0001$, respectively. The mean score of mothers with AN was again in the opposite direction although only 0.01 lower than the No ED group $(\mathrm{M}=1.24, \mathrm{SE}=0.003)$. Mean scores of mothers with $\mathrm{BN}$ and BED were 0.8 and 0.7 higher than the referent, respectively. Mothers with $\mathrm{BN}$ reported higher levels of OCD symptoms in their children than those with No ED, $p<.002$. In this instance, compared with the No ED group $(\mathrm{M}=1.28, \mathrm{SE}=0.003)$, scores were higher in the AN, BN and BED groups by $0.15,0.13$, and 0.04 , respectively. While reported infant depression significantly differed overall across eating disorder status, $\mathrm{F}(3,12856)=3.83, p<.009$, there were no differences across pairwise comparisons. Compared to the adjusted means for infant depression for the No ED group, women with AN had the same mean, and those with BN, and BED were . 03 and .01 higher. 


\subsection{Relation between Maternal-Report of Feeding and Child Eating Behavior}

To examine the relation between maternal reported feeding behaviors and child eating behavior, two models were created corresponding to restrictive feeding and pressured feeding. After adjusting for child food allergy and child weight-for-age status, maternally reported restrictive feeding was significantly associated with child disordered eating behavior in the positive direction, $\mathrm{F}(1,10640)=100.03, p<.0001$. A significant positive relation also emerged between pressured feeding and child eating problems, $\mathrm{F}(1,10625)=1970.09, p<.0001$.

\section{Discussion}

Little is known about how mothers with eating disorders feed their children, and there have been no prior prospective, large-scale, population-based cohorts of mothers with eating disorders that have examined this topic. We noted significant differences on self-reported restrictive maternal feeding behavior in women with some eating disorders relative to the noneating disorders referent group. Overall, patterns of restrictive feeding were more common in mothers with eating disorders marked by symptoms binge eating (i.e., BED and BN) than those with No ED. No differences emerged across eating disorder groups regarding pressured feeding.

An important question to address was the extent to which these observed differences were due primarily to differences in maternal BMI given the known differences in feeding styles associated with maternal overweight and obesity (Francis, Hofer, \& Birch, 2001; Johannsen, Johannsen, \& Specker, 2006). On average, women with BN and BED fell into the overweight category, whereas women with AN or No ED were in the healthy weight category. Maternal BMI did not appear to be the driving factor in our observations. Although significant as an independent predictor, its inclusion as a covariate did not diminish the impact of maternal eating disorders. Thus eating disorders appear to influence restrictive maternal feeding style independent of maternal BMI.

Overall, maternal $\mathrm{BN}$ and $\mathrm{BED}$ were associated with greater reports of child eating problems relative to the No ED group. This finding is important in beginning to elucidate differences in eating behaviors in children of mothers across eating disorders subtypes-differences which may increase as the child matures. Regarding no differences found on pressured feeding style across groups, this may be a feeding dimension that is unaltered by the presence of an eating disorder.

In terms of child psychological symptoms, significant differences emerged across eating disorders status on maternal reported child anxiety and OCD symptoms. Higher child anxiety symptoms were reported in children of mothers with BN and BED and mothers with BN reported higher OCD symptoms in their children than mothers without eating disorders. Elevated anxiety in offspring of women with eating disorders is consistent with observations of significantly elevated comorbidity of anxiety disorders in women with eating disorders (Brewerton, et al., 1995; Bulik, Sullivan, Carter, \& Joyce, 1996; Bulik, Sullivan, Fear, \& Joyce, 1997; Deep, Nagy, Weltzin, Rao, \& Kaye, 1995; Garfinkel, et al., 1995; Schwalberg, Barlow, Alger, \& Howard, 1992; Raney et al., 2008). The small AN sample size may again have precluded the detection of significant differences. Moreover, the children in the sample were only 36 months old, which is early for the emergence of anxiety and depressive psychopathology in children. Unexpectedly, although an overall significant effect was found, no significant differences occurred in pairwise comparisons of maternal-reported child depressive symptoms across eating disorders status. Whereas there is significant evidence for familial aggregation of depression in eating disorders (Hudson, Pope, Jonas, \& Yurgelun-Todd, 1983; Silberg \& Bulik, 2005), the lack of differences across groups may relate to the overall 
difficulty in capturing depression, an internalized (less visible) characteristic, in very young children (Klein, Dougherty, \& Olino, 2005).

Regarding the association between maternal feeding behavior and child eating behavior, restrictive and pressured feeding were both significantly associated with child disordered eating behaviors. Although the analyses were not limited to women with eating disorders, it is notable, although perhaps not surprising, that a relation exists between the way a mother feeds her child and the way the child eats. The direction of causality cannot be discerned from our design. It is critical to note that both measures were provided by maternal report and as such mothers who perceive and report their own concerning feeding styles may be more likely to notice and report disordered eating behavior in their children.

The study's strengths must be evaluated in concert with its limitations. First, as the majority of data were collected from self-report measures, the impact of demand characteristics on accuracy of report must be considered. Further, due to denial or normalization of eating disorders symptoms in women with AN, the potential for underreporting is possible. Second, the current study was limited in its ability to comprehensively assess current and lifetime symptom severity in individuals with eating disorders. Third, we were unable to collect information on children from multiple sources given the size of the sample. Fourth, the effect sizes of all significant findings were relatively small according to the conventions of Cohen (Cohen, 1988), suggesting that the impact of maternal feeding styles at this age might be relatively small. Fifth, the current sample had a somewhat low participation rate (42\%), although this does not necessarily assume bias. Finally, it must be noted that no significant differences were found relative to mothers with AN. However, differences in mean scores between the AN and No ED groups were equal to or larger in magnitude than the significant $\mathrm{BN}$ or BED differences on the majority of tests and often in the opposite direction. Whereas the lack of significant difference is due to the highly imbalanced sample sizes across groups, the opposite direction of results should nonetheless be considered. Several possibilities emerge including the possibility that women with AN who participated in the MoBa had relatively mild cases of AN with less physical sequelae, leading to more normative responses on outcome measures, supported by recent population data (Keski-Rahkonen, et al., 2007).

Alternatively, women with AN may be more likely to respond to demand characteristics and be less likely to report unhealthy eating and feeding patterns in their children. Finally, women with AN who are well enough to have children and participate in a study like MoBa might be fearful of transmitting unhealthy eating behaviors to their children and might strive particularly hard to create a healthy eating environment.

\subsection{Conclusions}

The current study confirms that reported restrictive maternal feeding behavior and child eating patterns significantly differ across eating disorders status. In particular, mothers with bingetype eating disorders may be more likely than other subgroups to endorse restrictive feeding styles. While pressured feeding was not found to differ significantly across groups, theoretically, maternal control associated with restrictive feeding could serve to override children's natural abilities to self-regulate intake (Birch \& Deysher, 1986) and ultimately, albeit inadvertently, contribute to the development of disordered eating.

These findings raise some concern. Although genetic factors contribute to eating disorders risk, environmental factors also play a role (Bulik, Sullivan, Wade, \& Kendler, 2000; Klump, et al., 2001; Klump et al., 2007; Wade, Bulik, Neale, \& Kendler, 2000). Maternal factors do play a considerable role in child development and eating behaviors (Essex, et al., 2006; Johannsen, et al., 2006; Pickles, et al., 1994). Offspring of mothers with eating disorders could conceivably be both inheriting risk alleles for disordered eating and be exposed to environmental factors 
(maternal feeding practices) which could unintentionally contribute to dysregulated eating. Additional in depth information would be of value assessing how in mothers with eating disorders think and feel about feeding their children and their actual ability to feed their children in a healthy way. However, although speculative, the current study's findings suggest that it is not premature to consider the potential value of educational programs and support for mothers with eating disorders. Indeed such early intervention could serve as a means by which we could interrupt the "cycle of risk" associated with eating disorders (Patel, et al., 2002; Russell, et al., 1998; Stein, et al., 2006).

\section{Acknowledgments}

This research was supported by the National Institutes of Health Grants (HD047186) to C.M.B. and the MoBa study is supported by the Norwegian Ministry of Health, NIH/NIEHS (grant no. N01 - ES - 85433), NIH/NINDS (grant no. 1 UO1 NS 047537-01) and Norwegian Research Council/FUGE (grant no. 151918/S10). L.R.H. was supported by a Ruth L. Kirschstein National Research Service Awards for Individual Predoctoral Fellows (F31 HD057719-01). We also thank the Norway-America Association, who provided a Marshall Fund travel grant to L.R.H. to collaborate with colleagues at the Norwegian Institutes of Public Health. The donation of questionnaire data and biological material from MoBa participants is gratefully acknowledged.

\section{Abbreviations}

$\begin{array}{ll}\text { AN } & \text { Anorexia nervosa } \\ \text { BN } & \text { Bulimia nervosa } \\ \text { BED } & \text { Binge eating disorder } \\ \text { No ED } & \text { No eating disorder }\end{array}$

\section{References}

Achenbach TM. National survey of problems and competencies among 4- to 16 years olds- Parents reports for normative and clinical samples. Monographs of the Society for Research in Child Development 1991;56:R5-R119.

Agras S, Hammer L, McNicholas F. A prospective study of the influence of eating disordered mothers on their children. International Journal of Eating Disorders 1999;25:253-262. [PubMed: 10191989]

Barr CS, Newman TK, Shannon C, Parker C, Dvoskin RL, Becker ML, et al. Rearing condition and rh5HTTLPR interact to influence limbic-hypothalamic-pituitary-adrenal axis response to stress in infant macaques. Biological Psychiatry 2004;55:733-738. [PubMed: 15039002]

Birch LL, Deysher M. Caloric compensation and sensory satiety: evidence for self-regulation of food intake by young children. Appetite 1986;7:323-331. [PubMed: 3789709]

Birch LL, Fisher JO, Grimm-Thomas K, Markey CN, Sawyer R, Johnson SL. Confirmatory factor analysis of the Child Feeding Questionnaire: a measure of parental attitudes, beliefs and practices about child feeding and obesity proneness. Appetite 2001;26:201-210. [PubMed: 11358344]

Brewerton T, Lydiard R, Herzog D, Brotman A, O'Neil P, Ballenger J. Comorbidity of Axis I psychiatric disorders in bulimia nervosa. Journal of Clinical Psychology 1995;56:77-80.

Bulik CM, Hebebrand J, Keski-Rahkonen A, Klump KL, Reichborn-Kjennerud T, Mazzeo SE, et al. Genetic epidemiology, endophenotypes, and eating disorder classification. International Journal of Eating Disorders 2007;40:S52-S60. [PubMed: 17573683]

Bulik CM, Sullivan PF, Carter F, Joyce P. Lifetime anxiety disorders in women with bulimia nervosa. Comprehensive Psychiatry 1996;37:368-374. [PubMed: 8879912]

Bulik CM, Sullivan PF, Fear J, Joyce P. Eating disorders and antecedent anxiety disorders: A controlled study. Acta Psychiatrica Scandinavica 1997;96:101-107. [PubMed: 9272193]

Bulik CM, Sullivan PF, Kendler KS. Genetic and environmental contributions to obesity and binge eating. International Journal of Eating Disorders 2003;33:293-298. [PubMed: 12655626] 
Bulik CM, Sullivan PF, Wade TD, Kendler K. Twin studies of eating disorders: a review. International Journal of Eating Disorders 2000;27:1-20. [PubMed: 10590444]

Bulik CM, Von Holle A, Hamer R, Knoph Berg C, Torgersen L, Magnus P, et al. Patterns of remission, continuation, and incidence of broadly defined eating disorders during early pregnancy in the Norwegian Mother and Child Cohort Study (MoBa). Psychological Medicine 2007;37:1109-1118. [PubMed: 17493296]

Carter AS, Briggs-Gowan MJ, Jones SM, Little TD. The Infant-Toddler Social and Emotional Assessment (ITSEA): factor structure, reliability, and validity. Journal of Abnormal Child Psychoogy 2003;31:495-514.

Carter AS, Little C, Briggs-Gowan MJ, Kogan N. The infant-toddler social and emotional assessment (ITSEA): Comparing parent ratings to laboratory observations of task mastery, emotion regulation, coping behaviours, and attachment status. Infant Mental Health Journal 1999;20:375-392.

Cohen, J. Statistical power analysis for the behavioral sciences. Hillsdale, NJ: Lawrence Erlbaum Associates; 1988.

Deep A, Nagy L, Weltzin T, Rao R, Kaye W. Premorbid onset of psychopathology in long-term recovered anorexia nervosa. International Journal of Eating Disorders 1995;17:291-298. [PubMed: 7773266]

Essex MJ, Kraemer HC, Armstrong JM, Boyce WT, Goldsmith HH, Klein MH, et al. Exploring risk factors for the emergence of children's mental health problems. Archives of General Psychiatry 2006;63:1246-1256. [PubMed: 17088505]

Evans J, le Grange D. Body size and parenting in eating disorders: a comparative study of the attitudes of mothers toward their children. International Journal of Eating Disorders 1995;18:39-48. [PubMed: 7670442]

Faul F, Erdfelder E, Lang A-G, Buchner A. G*Power 3: A flexible statistical power analysis program for the social, behavioral, and biomedical sciences. Behavior Research Methods. (in press)

Finster M, Wood M. The Apgar score has survived the test of time. Anesthesiology 2005;102:855-857. [PubMed: 15791116]

Francis LA, Hofer SM, Birch LL. Predictors of maternal child-feeding style: maternal and child characteristics. Appetite 2001;37(3):231-243. [PubMed: 11895324]

Franzen U, Gerlinghoff M. Parenting by patients with eating disorders: Experiences with a mother-child group. Eating Disorders 1997;5:5-14.

Garfinkel P, Lin E, Goering P, Spegg C, Goldbloom D, Kennedy S, et al. Bulimia nervosa in a Canadian community sample: prevalence and comparison of subgroups. American Journal of Psychiatry 1995;152:1052-1058. [PubMed: 7793442]

Hartge P. Participation in population studies. Epidemiology 2006;17:252-254. [PubMed: 16617271]

Hudson JI, Pope H, Jonas J, Yurgelun-Todd D. Phenomenologic relationship of eating disorders to major affective disorder. Psychiatry Research 1983;9:345-354. [PubMed: 6580663]

Johannsen DL, Johannsen NM, Specker BL. Influence of parents' eating behaviors and child feeding practices on children's weight status. Obesity (Silver Spring) 2006;14:431-439. [PubMed: 16648614]

Johnson J, Cohen P, Kasen S, Brook J. Childhood adversities associated with risk for eating disorders or weight problems during adolescence or early adulthood. American Journal of Psychiatry 2002;159:394-400. [PubMed: 11870002]

Keski-Rahkonen A, Hoek HW, Susser ES, Linna MS, Sihvola E, Raevuori A, et al. Epidemiology and course of anorexia nervosa in the community. American Journal of Psychiatry 2007;164:1259-1265. [PubMed: 17671290]

Klein DN, Dougherty LR, Olino TM. Toward Guidelines for Evidence-Based Assessment of Depression in Children and Adolescents. Journal of Clinical Child and Adolescent Psychology 2005;34:412432. [PubMed: 16026212]

Klump KL, Burt SA, McGue M, Iacono WG. Changes in genetic and environmental influences on disordered eating across adolescence: a longitudinal twin study. Archives of General Psychiatry 2007;64:1409-1415. [PubMed: 18056549]

Klump KL, Miller KB, Keel PK, McGue M, Iacono WG. Genetic and environmental influences on anorexia nervosa syndromes in a population-based twin sample. Psychological Medicine 2001;31:737-740. [PubMed: 11352375] 
Lacey J, Smith G. Bulimia nervosa: the impact of pregnancy on mother and baby. British Journal of Psychiatry 1987;150:777. [PubMed: 3651730]

Magnus P, Irgens LM, Haug K, Nystad W, Skaerven R, Stoltenberg C, et al. Cohort profile: The Norwegian Mother and Child Cohort Study (MoBa). International Journal of Epidemiology 2006;35:1146-1150. [PubMed: 16926217]

O’Reilly, D. Gestational Age. 2007 [Retrieved February 20, 2008]. from http://www.nlm.nih.gov/medlineplus/ency/article/002367.htm

Patel P, Wheatcroft R, Park RJ, Stein A. The children of mothers with eating disorders. Clinical Child Family Psychology Review 2002;5:1-19.

Pickles A, Neale M, Simonoff E, Rutter M, Hewitt J, Meyer J, et al. A simple method for censored ageof-onset data subject to recall bias: mothers' reports of age of puberty in male twins. Behavioral Genetics 1994;24:457-468.

Raney TJ, Thornton LM, Berrettini W, Brandt H, Crawford S, Fichter MM, Halmi KA, Johnson C, Kaplan AS, LaVia M, Mitchell J, Rotondo A, Strober M, Woodside DB, Kaye WH, Bulik CM. Influence of overanxious disorder of childhood on the expression of anorexia nervosa. International Journal of Eating Disorders 2008;41:326-332. [PubMed: 18213688]

Reichborn-Kjennerud T, Bulik CM, Kendler KS, Maes H, Roysamb E, Tambs K, et al. Gender differences in binge-eating: A population-based twin study. Acta Psychiatrica Scandinavica 2003;108:196-202. [PubMed: 12890274]

Reichborn-Kjennerud T, Bulik CM, Tambs K, Harris JR. Genetic and environmental influences on bingeeating in the absence of compensatory behaviors: a population- based twin study. International Journal of Eating Disorders 2004;36:307-314. [PubMed: 15478129]

Russell GF, Treasure J, Eisler I. Mothers with anorexia who underfeed their children: their recognition and management. Psychological Medicine 1998;28

SAS Institute Inc.. JMP User's Guide. (Version Version 3). Cary, S.C.: 1994.

SAS Institute Inc.. SAS/STAT® software: Version 9.1 (Version 9.1.3). Cary, NC: 2000-2004.

Schwalberg M, Barlow B, Alger S, Howard L. Comparison of bulimics, obese binge eaters, social phobics, and individuals with panic disorder on comorbidity across DSM-III-R anxiety disorders. Journal of Abnormal Psychology 1992;101:675-681. [PubMed: 1430607]

Silberg J, Bulik CM. The developmental association between eating disorders symptoms and symptoms of anxiety and depression in juvenile twin girls. Journal of Child Psychoology andl Psychiatry 2005;46:1317-1326.

Stein A, Woolley H, Cooper S, Winterbottom J, Fairburn CG, Cortina-Borja M. Eating habits and attitudes among 10-year-old children of mothers with eating disorders: Longitudinal study. British Journal of Psychiatry 2006;189:324-329. [PubMed: 17012655]

Wade TD, Bulik CM, Neale M, Kendler KS. Anorexia nervosa and major depression: shared genetic and environmental risk factors. American Journal of Psychiatry 2000;157:469-471. [PubMed: 10698830] 


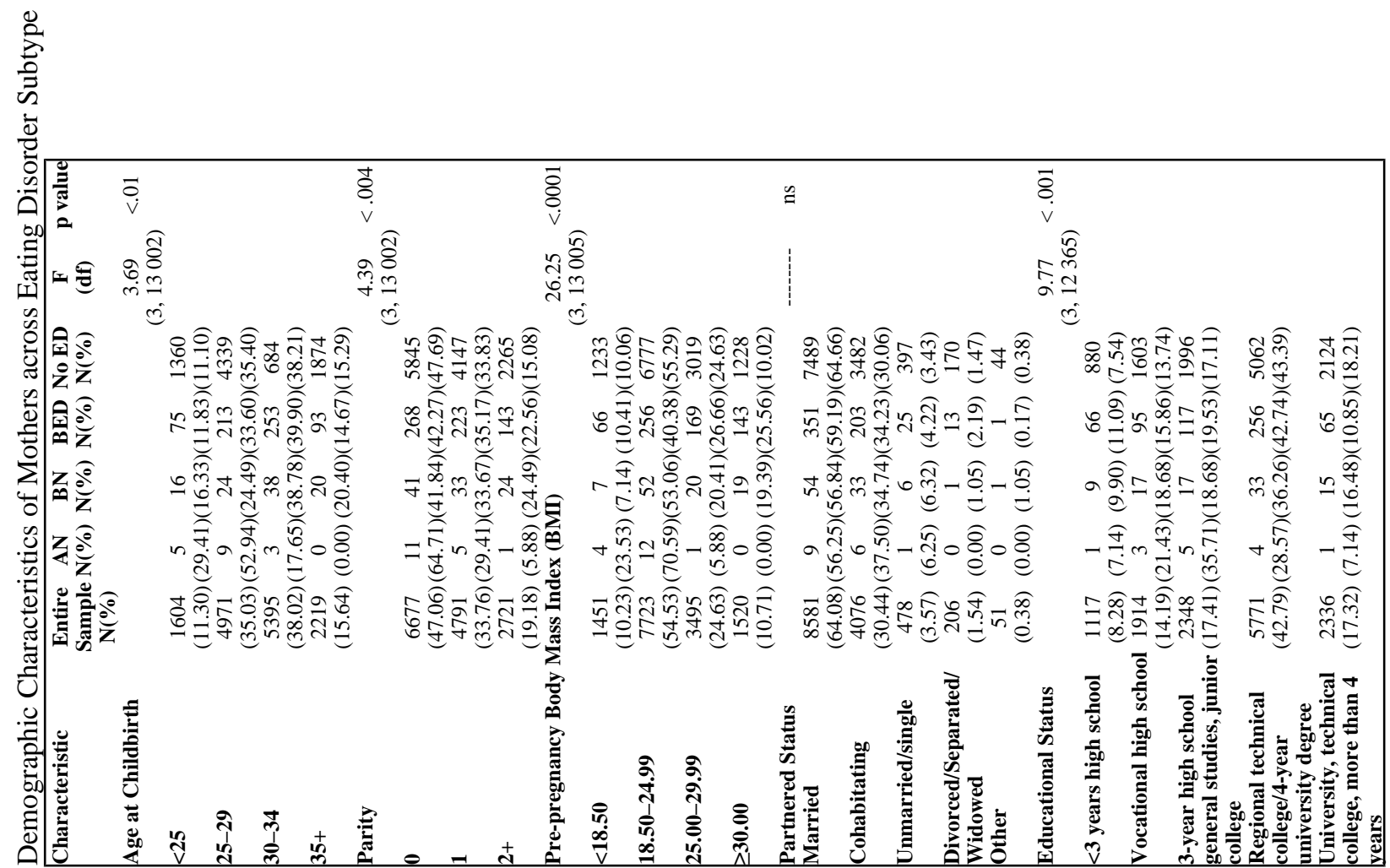




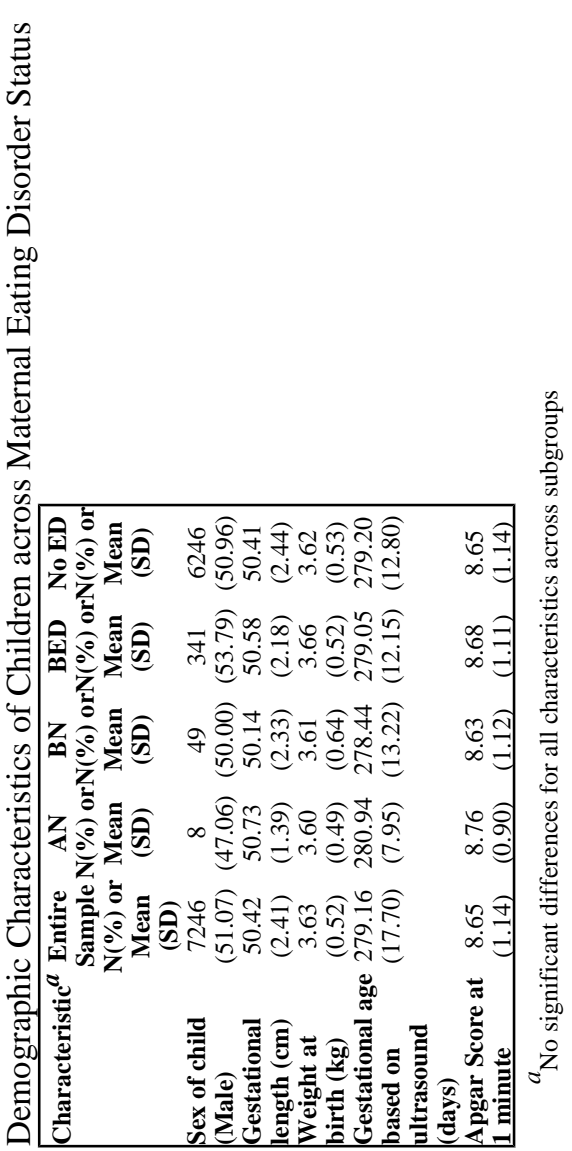

Eat Behav. Author manuscript; available in PMC 2011 January 1. 


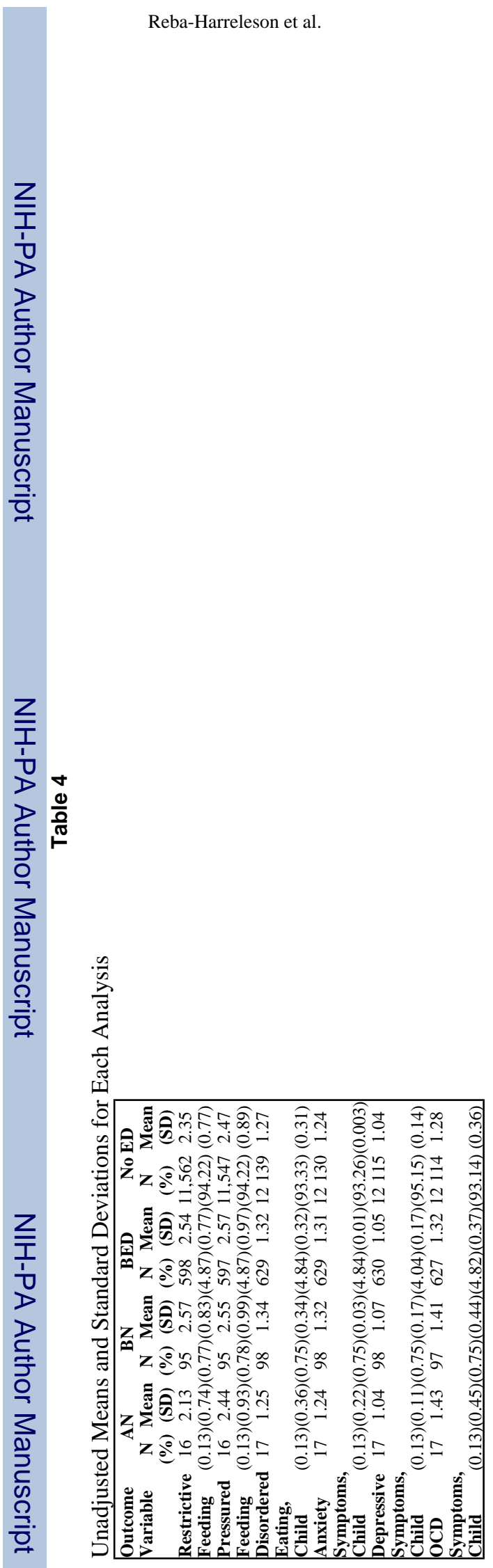

Page 16

Eat Behav. Author manuscript; available in PMC 2011 January 1. 